\title{
Blood pressure variability in primary hyperparathyroidism: more data needed
}

\author{
R. Antonelli ${ }^{1}{ }^{1}$
}

Received: 24 October 2017 / Accepted: 8 November 2017 / Published online: 20 November 2017

(C) Springer Science+Business Media, LLC, part of Springer Nature 2017

We read with interest the article recently published on line by Concistrè [1] and co-workers. The authors conclude in their abstract that blood pressure variability is increased in normotensive patients with primary hyperparathyroidism and is reduced by parathyroidectomy. However, no specific changes of normotensive hyperparathyroid patients following surgery is reported in the results sections. Then, according to the study population investigated, these patients should be only 5 , a very small sample to draw definitive conclusions. Secondly, while operated patients were re-evaluated after a follow-up time of $1.9 \pm 1.1$ years, nothing is said about time elapsed since the first evaluation in non-operated patients. This point is of utmost importance; indeed, it appears that some of the parameters (i.e., 24-h SD of systolic blood pressure, 24-h weighted SD of systolic blood pressure) have an important decrease, even though statistical significance is not reported. Finally, the authors argue that 24-h average real variability is directly dependent on serum calcium and PTH values (Figure 3). However, this hypothesis is derived by merging hypertensive (with normal serum calcium and parathyroid hormone values) with hyperparathyroid (with high serum calcium and parathyroid hormone values) patients. We believe that this is not a good statistical approach to validate their hypothesis. Indeed, inspection of the figure seems to show that such a correlation hardly persists, only considering hyperparathyroid patients, whether hypertensive or not. We believe that more robust data are therefore needed to reach their conclusions.

\section{Compliance with ethical standards}

Conflict of interest The author declares that she has no competing interests

Ethical approval This study does not contain any study with human participant

\section{Reference}

1. A. Concistrè, A. Grillo, G. La Torre et al., Ambulatory blood pressure monitoring-derived short-term blood pressure variability in primary hyperparathyroidism Endocrine (2017). https://doi.org/ 10.1007/s12020-017-1362-x
R. Antonelli

rosantonelli@tiscali.it

1 Osp. S.Eugenio \& Osp.CTO, Roma, Italy 